\title{
Medizinische Informationsflut und Wissenstransfer in die Praxis - eine Quadratur des Kreises?
}

\author{
Medizinisches Wissen ist entscheidend für eine optimale Krankenversorgung. \\ Dieses soll in Studium, Facharztausbildung und kontinuierlicher Fortbildung \\ erlernt und aktualisiert werden, um eine Behandlung nach aktuellsten wissen- \\ schaftlichen Erkenntnissen zu gewährleisten. Doch wie sieht die Realität aus?
}

\section{A. Sönnichsen, A. Rinnerberger}

Korrespondenz:

Prof. Dr. Andreas Sönnichsen Paracelsus Medizinische

Privatuniversität

Strubergasse 21

A-5020 Salzburg

www.pmu.ac.at

\section{Publikationen pro Monat}

Eine Studie zeigte, dass das Wissen von Internisten kontinuierlich abnimmt. 15 Jahre nach der Facharztprüfung würden diese nur noch $30 \%$ der Ärzte bestehen [1]. Nur etwa $40 \%$ aller Patienten werden nach aktuellen Leitlinien versorgt [2]. Bis zu $25 \%$ der Patienten wird durch eine nicht indizierte Behandlung Schaden zugefügt [3, 4]. Und die Zunahme von Wissensdefiziten scheint vorprogrammiert: Waren die Ärzte 1988 noch mit nur etwa 30000 monatlich neu erscheinenden Publikationen konfrontiert, so sind es heute 64000 . Die Anzahl der Neuerscheinungen hat sich also in den letzten zwei Jahrzehnten mehr als verdoppelt.

Selbst die explizite Suche nach klinischen Studien ergibt für 2007 noch die stattliche Zahl von etwa 3000 Publikationen pro Monat! Es wird geschätzt, dass ein Allgemeinarzt täglich etwa 20 Originalarbeiten lesen müsste, um einigermassen auf dem Laufenden zu bleiben [5], dies unter der Annahme, dass nur 20\% der Arbeiten tatsächlich klinische Relevanz besitzen.

Es stellt sich also die Frage, wie wir mit der Flut an medizinischer Information, die täglich über uns hereinbricht, umgehen. Es gibt verschiedene Lösungsansätze. Einer ist die Kondensation von Informationen zu systematischen Reviews und Leitlinien. Hier hat sich die internationale Cochrane Collaboration (www.cochrane.org) grösste Verdienste erworben. Medizinische Fachliteratur wird nach Evidenz durchforstet und als Reviews in der Cochrane Library zugänglich gemacht (www.mrw.interscience.wiley.com/ cochrane). Doch wollte man daran gehen, sich das so zusammengetragene Wissen zu eigen zu machen, so stünde man vor einem unüberwindbaren Berg von heute bereits 6440 Reviews. Diese - im übrigen in der Schweiz, in Österreich und in Deutschland kostenpflichtige - Daten- bank kommt also nur als Nachschlagewerk bei konkreten Fragen in Betracht und deckt nur einen Bruchteil der in der täglichen Praxis auftauchenden Probleme ab.

Etwas umfassender und handlicher, aber weniger akribisch als Cochrane, sind die EBMGuidelines für Allgemeinmedizin, die als Buchund Onlineversion zur Verfügung stehen [6]. Eine besonders komfortable Lösung stellt ein

\section{FORUM MEDIZIN 21}

Ärztin/Arzt sein im 21. Jahrhundert: Erwartung - Selbstbild - Realität

Vom 29. bis 31. Januar 2009 findet in Salzburg/A das Forum Medizin 21 statt, ein international ausgerichteter Kongress, der auch für Schweizer Ärztinnen und Ärzte interessant ist. Besonderes Augenmerk wird auf den Wissenstransfer zwischen Wissenschaft und Praxis, auf die Diskussion aktueller Themen sowie auf den persönlichen Austausch gelegt. Die drei Kongresstage sind den Themen «Information und Informationsflut», «Gesundheit und Ökonomie» und «ArztPatienten-Beziehung heute» gewidmet. Da die Wechselwirkung zwischen Ausbildung und Beruf gerade in der Medizin von hoher Relevanz ist, soll das Forum auch Impulse für die Aus-, Weiter- und Fortbildung geben.

Die Veranstaltung ist von der Österreichischen Ärztekammer mit 18 Punkten (freie Fortbildung) bewertet. Diese können auch in der Schweiz berücksichtigt werden ( 1 Stunde $=1$ Credit). Auskunft geben die Fachgesellschaften.

Unter www.forummedizin21.at finden Interessierte weiterführende Informationen und Anmeldemodalitäten. 
Rechercheservice dar: Der Arzt schickt Fragen aus der Praxis über das Internet an ein Rechercheteam und erhält eine evidenzbasierte Lösung [7].

Doch all diese Möglichkeiten, aus der Flut medizinischer Information Relevantes zu extrahieren, haben eines gemein: Sie erfordern das Bewusstsein des Arztes, dass ein Wissensdefizit vorliegt. Im medizinischen Alltag - und nicht nur dort - haben wir es aber mit zwei Formen des Nichtwissens zu tun, die der Schweizer Arzt M. Deppeler so dargestellt hat [8]:

- Ich weiss nicht, dass ich etwas nicht weiss;

- Ich weiss, dass ich etwas nicht weiss.

Die erste Form des Nichtwissens ist (nicht nur) unter Ärzten wesentlich verbreiteter als die zweite. Der Wissenstransfer in die Praxis scheitert also oft nicht nur an der Informationsflut, sondern vor allem auch am Bewusstsein der Ärzte für die Notwendigkeit, ihr bestehendes Wissen $\mathrm{zu}$ hinterfragen. Alle bisher evaluierten Strategien zur Implementierung von aktuellem Wissen, seien es nun CME, schriftliche Informationen, Fortbildungsveranstaltungen usw. zeigten nur limitierte Erfolge [9].

Die derzeit vorliegenden Defizite in der Patientenversorgung machen deutlich, wie wichtig die Thematik ist. Mit diesen und weiteren Herausforderungen des Arztberufes setzt sich die Paracelsus Medizinische Privatuniversität Salzburg im Januar 2009 im Rahmen des ersten «Forum Medizin 21» auseinander. Unter dem Titel «Ärz-
tin/Arzt sein im 21. Jahrhundert» sollen unter anderem Strategien für einen möglichen Umgang mit der Informationsflut entwickelt werden.

\section{Literatur}

1 Ramsey PG, Carline JD, Inui TS, Larson EB, LoGerfo JP, Norcini JJ, et al. Changes over time in the knowledge base of practicing internists. JAMA. 1991;266(8):1103-7.

2 Freemantle N, Nazareth I, Eccles M, Wood J, Haines A. A randomised controlled trial of the effect of educational outreach by community pharmacists on prescribing in UK general practice. Br J Gen Pract. 2002;52(477):290-5.

3 Schuster MA, McGlynn EA, Brook RH. How good is the quality of health care in the United States? Milbank Q. 1998;76(4):517-63, 509.

4 Grol R. Successes and failures in the implementation of evidence-based guidelines for clinical practice. Med Care. 2001;39(8 Suppl 2):II46-II54.

5 Shaneyfelt TM. Building bridges to quality. JAMA. 2001;286(20):2600-1.

6 Rabady S, Rebhandl E, Sönnichsen A. EBM-Guidelines für Klinik und Praxis. 4. Auflage. Basel: EMH Schweizerischer Ärzteverlag; 2008.

7 Rinnerberger A, Url MG, Sönnichsen A. Rechercheservice Evidenzbasierte Medizin (REM) Pilotprojekt zur Förderung evidenzbasierter Medizin in der hausärztlichen Versorgung. Zeitschrift für Allgemeinmedizin. 2007;83(S):20.

8 Deppeler M. Denn sie wissen (nicht), was sie tun. PrimaryCare. 2007;7(42):646-8.

9 Grol R, Grimshaw J. From best evidence to best practice: effective implementation of change in patients' care. Lancet. 2003;362(9391):1225-30. 